\title{
Odd Graceful Labeling of Acyclic Graphs
}

\author{
Ayesha Riasat ${ }^{1}$, Sana Javed ${ }^{2}$ \\ ${ }^{1}$ Mathematics Department, University of Management and Technology, Lahore, Pakistan \\ ${ }^{2}$ Mathematics Department, Comsats Institute of information Technology, Lahore, Pakistan \\ Email address: \\ ayeshaa.riasat@gmail.com (A. Riasat), unique.sana@hotmail.com (S. Javed)
}

\section{To cite this article:}

Ayesha Riasat, Sana Javed. Odd Graceful Labeling of Acyclic Graphs. American Journal of Applied Mathematics. Special Issue: Proceedings of the 1st UMT National Conference on Pure and Applied Mathematics (1st UNCPAM 2015). Vol. 3, No. 3-1, 2015, pp. 14-18.

doi: $10.11648 /$ j.ajam.s.2015030301.13

\begin{abstract}
Let $\mathrm{G}=(\mathrm{V}, \mathrm{E})$ be a finite, simple and undirected graph. A graph $\mathrm{G}$ with q edges is said to be odd-graceful if there is an injection $\mathrm{f}: \mathrm{V}(\mathrm{G}) \rightarrow\{0,1,2, \ldots, 2 \mathrm{q}-1\}$ such that, when each edge xy is assigned the label $|\mathrm{f}(\mathrm{x})-\mathrm{f}(\mathrm{y})|$, the resulting edge labels are $\{1,3,5, \ldots, 2 \mathrm{q}-1\}$ and $\mathrm{f}$ is called an odd graceful labeling of G. Motivated by the work of Z. Gao [6] in which he studied the odd graceful labeling of union of any number of paths and union of any number of stars, we have determined odd graceful labeling for some other union of graphs. In this paper we formulate odd-graceful labeling for disjoint unions of graphs consisting of generalized combs, stars, bistars and paths.
\end{abstract}

Keywords: Odd-Graceful Labeling, Comb, Star, Path, Bistar

\section{Introduction}

Most graph labeling methods trace their origin to one introduced by Rosa [12] in 1967, or one given by Graham and Sloane [9] in 1980. Rosa [12] called a function $f$ a $\beta$. valuation of a graph $G$ with $q$ edges if $f$ is an injection from the vertices of $G$ to the set $\{0,1, \ldots, q\}$ such that, when each edge $x y$ is assigned the label $|f(x)-f(y)|$, the resulting edge labels are distinct. Golomb [8] subsequently called such labeling graceful and this is now the popular term. Rosa introduced $\beta$-valuations as well as a number of other labeling as tools for decomposing the complete graph into isomorphic subgraphs. In particular, $\beta$-valuations originated as a mean of attacking the conjecture of Ringel [11] that $K_{2 n+1}$ can be decomposed into $2 n+1$ subgraphs that are all isomorphic to a given tree with $n$ edges.

When a graceful labeling $f$ of a graph $G$ has the property that there exists an integer $\lambda$ such that for each edge $x y$ of $G$ either $f(x) \leq \lambda<f(y)$ or $f(y) \leq \lambda<f(x), f$ is named an $\alpha$-labeling and $G$ is said to be an $\alpha$-graph. From the definition it is possible to deduce that a $\alpha$-graph is necessarily bipartite and that the number $\lambda$ (called the boundary value of $f$ ) is the smaller of the two vertex labels that yield the edge with weight 1 . Some examples of $\alpha$ graphs are the cycle $C_{n}$ when $n \equiv 0(\bmod 4)$, the complete bipartite graph $K_{m, n}$, and caterpillars (i.e., any tree with the property that the removal of its end vertices leaves a path). For detailed studies one can see [2] and [1].

A little less restrictive than $\alpha$-labeling are the oddgraceful labeling introduced by Gnanajothi in 1991 [7]. A graph $G$ of size $n$ is odd-graceful if there is an injection $f: V(G) \rightarrow\{0,1,2, \ldots, 2 n-1\}$ such that the set of induced weights is $\{1,3, \ldots, 2 n-1\}$. In this case, $f$ is said to be an odd-graceful labeling of $G$. One of the applications of this labeling is that trees of size $n$, with a suitable odd-graceful labeling, can be used to generate cyclic decompositions of the complete bipartite graph $K_{n, n}$.

In general graph labeling serves as useful model for a broad range of applications such as: radar, communications network, circuit design, coding theory, astronomy, x-ray, crystallography, data base management and models for constraint programming over finite domains.

Gnanajothi proved that the class of odd-graceful graphs lies between the class of graphs with $\alpha$-labeling and the class of bipartite graphs by showing that every graph with an $\alpha$-labeling has an odd-graceful labeling and every graph with an odd cycle is not odd-graceful. She also proved the following graphs are odd-graceful: $P_{n}, C_{n}$ if and only if $n$ is even, $K_{m, n}$ and combs.

She conjectured that all trees are odd-graceful and proved the conjecture for all trees with order up to 10 . Barrientos [3] 
has extended this to trees of order up to 12 . Eldergill [4] generalized Gnanajothi's result on stars by showing that the graphs obtained by joining one end point from each of any odd number of paths of equal length are odd-graceful.

A detailed account of results in the subject of graph labelings can be found in Gallian' survey [5].

Gao [6] has proved the following graphs are odd-graceful: the union of any number of paths; the union of any number of stars; the union of any number of stars and paths; $C_{m} \cup P_{n}$; $C_{m} \cup C_{n}$; and the union of any number of cycles each of which has order divisible by 4 .

In [10] authors have found odd graceful labeling of $C b_{n}(k, k, \ldots, k)$ and $C b_{n}(2,3, \ldots, n+1)$ for $n \geq 2$ and $k \geq 3$. Further odd graceful labeling of two copies of generalized combs are discussed along with their disjoint union with other well known families of graphs, also an open problem was proposed to find the odd graceful labeling of union of $C b_{n}(2,3, \ldots, n+1)$ with $B_{l, m}$ for $l \neq m$. Motivated by this open problem in [10], in this paper we have defined odd graceful labeling for $C b_{n}(2,3, \ldots, n+1)$ union $B_{l, m}$ for $l \neq m$. We also formulate an odd graceful labeling of the union of $C b_{n}(k, k, \ldots, k), C b_{n}(2,3, \ldots, n+1)$ with paths and stars.

\subsection{Definition 1}

A generalized comb is a graph derived from the path $P_{n+1}: x_{1,0}, x_{1,1}, \ldots, x_{1, n}, n \geq 2$ by adding $n$ new paths $x_{2, j}, x_{3, j}, \ldots, x_{k_{i}, j}$ of lengths $k_{i}-2$, where $k_{i} \geq 2,1 \leq i \leq n$ and new edges $x_{1, j} x_{2, j}$ for $1 \leq j \leq n$ (see Fig. 1 ) and this is denoted by $C b_{n}\left(k_{1}, k_{2}, \ldots, k_{n}\right) . C b_{n}(2,2, \ldots, 2)$ is simply called a comb and it is denoted by $C b_{n}$.

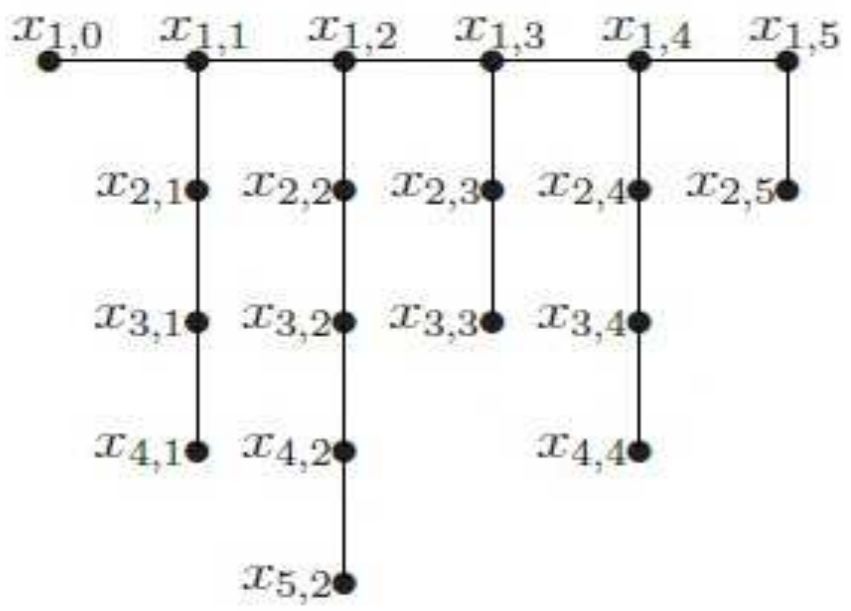

Figure 1. $C b_{s}(4,5,3,4,2)$

\subsection{Definition 2}

A star is a tree with a central node $y_{0}$ and $l$ leaves $\left\{y_{1}, y_{2}, \ldots, y_{l}\right\}$ adjacent to $y_{0}$. A star is a complete bipartite graph $k_{1, l}$.

\subsection{Definition 3}

A bistar $B_{m, l}$ is obtained by joining central nodes $y_{0}$ and $z_{0}$ of two stars $S_{l}$ and $S_{m}$ respectively, by an edge.

\section{Odd Graceful Labeling of Disjoint Union of Generalized Combs with Stars, Bistars and Paths}

This section describes odd graceful labeling for union of generalized combs with stars, bistars and paths.

Theorem $2.1 C b_{n}(k, k, \ldots, k) \cup P_{m}$ is odd graceful for $n, m \geq 2$ and $k \geq 3$.

Proof. Let $G \cong C b_{n}(k, k, \ldots, k) \cup P_{m}$. Then we denote vertex set and edge set of graph $G$ as follows:

$$
\begin{gathered}
V(G)=\left\{x_{i, j}: 1 \leq i \leq k, 1 \leq j \leq n\right\} \cup\left\{x_{1,0}\right\} \cup\left\{y_{i}: 1 \leq i \leq m\right\}, \\
E(G)=\left\{x_{i, j} x_{i+1, j}: 1 \leq i \leq k-1,1 \leq j \leq n\right\} \cup \\
\left\{x_{1, j} x_{1, j+1}: 0 \leq j \leq n-1\right\} \cup\left\{y_{i} y_{i+1}: 1 \leq i \leq m-1\right\}
\end{gathered}
$$

Where $|V(G)|=n k+m+1$ and $q=|E(G)|=n k+m-1$. We define the labeling $f: V(G) \rightarrow\{0,1,2, \ldots, 2 q-1\}$. Case (i)

For odd $n$ where $n, k \geq 3$ and $m \geq 2$

$$
f\left(x_{1,0}\right)=(n-1) k+2 m-1
$$

For $1 \leq i \leq k, 1 \leq j \leq n$

$$
f\left(x_{i, j}\right)=\left\{\begin{array}{lr}
(n+j) k+2 m-i-1 & \text { if } i \equiv 0(\bmod 2), \\
& j \equiv 1(\bmod 2) ; \\
(n+j-1) k+2 m+i-2 & \text { if } i \equiv 1(\bmod 2), \\
(n-j) k+i-1 & j \equiv 0(\bmod 2) ;(\bmod 2), \\
& j \equiv 1(\bmod 2) ; \\
(n-j+1) k-i & \text { if } i \equiv 0(\bmod 2), \\
& j \equiv 0(\bmod 2) .
\end{array}\right.
$$

For $1 \leq i \leq m$

$$
f\left(y_{i}\right)= \begin{cases}(n-1) k+2 m+i-3 & \text { if } i \equiv 1(\bmod 2) \\ (n-1) k+2 m-i-5 & \text { if } i \equiv 0(\bmod 2) .\end{cases}
$$

Case (ii)

For even $n$ where $k \geq 3, n, m \geq 2$ and

$$
f\left(x_{1,0}\right)=n k
$$

For $1 \leq i \leq k, 1 \leq j \leq n$ 


$$
f\left(x_{i, j}\right)=\left\{\begin{array}{lr}
(n-j+1) k-i & \text { if } i \equiv 0(\bmod 2), \\
& j \equiv 1(\bmod 2) ; \\
& \text { if } i \equiv 1(\bmod 2), \\
(n-j) k+i-1 & j \equiv 0(\bmod 2) ; \\
(n+j-1) k+2(m-1)+i & \text { if } i \equiv 1(\bmod 2), \\
& j \equiv 1(\bmod 2) ; \\
(n+j) k+2 m-i-1 & \text { if } i \equiv 0(\bmod 2), \\
& j \equiv 0(\bmod 2) .
\end{array}\right.
$$

For $1 \leq i \leq m$

$$
f\left(y_{i}\right)= \begin{cases}n k+2 m+i-3 & \text { if } i \equiv 1(\bmod 2) \\ n k+2 m-i-1 & \text { if } i \equiv 0(\bmod 2) .\end{cases}
$$

We can see that the set of edge weights $\{1,3,5, \ldots, 2 q-1\}$, where $2 q-1=2(n k+m)-3$ consists of $q$ consecutive odd integers. Therefore $f$ is an odd graceful labeling of $G$.

Theorem $2.2 C b_{n}(k, k, \ldots, k) \cup B_{m, l}$ for $m=l$ has odd graceful labeling where $n, m, l \geq 2$ and $k \geq 3$.

Proof. Consider $G \cong C b_{n}(k, k, \ldots, k) \cup B_{m, l}$. We have $|V(G)|=n k+m+l+3$ and $q=|E(G)|=n k+m+l+1$, where

$$
\begin{aligned}
& V(G)=\left\{x_{i, j}: 1 \leq i \leq k, 1 \leq j \leq n\right\} \cup\left\{x_{1,0}\right\} \cup\left\{z_{i}: 0 \leq i \leq m\right\} \cup \\
& \left\{y_{i}: 0 \leq i \leq l\right\}
\end{aligned}
$$

and

$$
\begin{aligned}
& E(G)=\left\{x_{i, j} x_{i+1, j}: 1 \leq i \leq k-1,1 \leq j \leq n\right\} \cup \\
& \left\{x_{1, j} x_{1, j+1}: 0 \leq j \leq n-1\right\} \cup\left\{z_{0} z_{i}: 1 \leq i \leq m\right\} \cup \\
& \left\{y_{0} y_{i}: 1 \leq i \leq l\right\} \cup\left\{y_{0} z_{0}\right\} .
\end{aligned}
$$

To show that $G$ is odd graceful we define the labeling $f: V(G) \rightarrow\{0,1,2, \ldots, 2 q-1\}$.

For $n, m, l \geq 2, k \geq 3$ and $m=l$

For $0 \leq i \leq m$

$$
f\left(z_{i}\right)= \begin{cases}i & \text { if } 0 \leq i \leq 1 \\ 2 i-1 & \text { if } 2 \leq i \leq m .\end{cases}
$$

For $0 \leq i \leq l$

$$
f\left(y_{i}\right)= \begin{cases}2(m+l+n k)+1 & \text { if } i=0 \\ 2 i & \text { if } 1 \leq i \leq l .\end{cases}
$$

\section{Case $(i)$}

For odd $n$ where $n, k \geq 3$ and $l, m \geq 2$

$$
f\left(x_{1,0}\right)=(n+1) k+2(l+m+1)
$$

$$
\begin{aligned}
& \text { For } 1 \leq i \leq k, 1 \leq j \leq n \\
& f\left(x_{i, j}\right)=\left\{\begin{array}{lr}
(n+j) k+2(l+m)-i+2 & \text { if } i \equiv 0(\bmod 2), \\
& j \equiv 1(\bmod 2) ; \\
(n+j-1) k+2(l+m)+i+1 & \text { if } i \equiv 1(\bmod 2), \\
& j \equiv 0(\bmod 2) ; \\
(n-j) k+i+l+m & \text { if } i \equiv 1(\bmod 2), \\
& j \equiv 1(\bmod 2) ; \\
(n-j+1) k-i+l+m+1 & \text { if } i \equiv 0(\bmod 2), \\
& j \equiv 0(\bmod 2) .
\end{array}\right.
\end{aligned}
$$

Case(ii)

For even $n$ where $k \geq 3, n, m, l \geq 2$ and

$$
f\left(x_{1,0}\right)=n k+l+m+1
$$

For $1 \leq i \leq k, 1 \leq j \leq n$

$$
f\left(x_{i, j}\right)=\left\{\begin{array}{lr}
(n-j+1) k+l+m-i+1 & \text { if } i \equiv 0(\bmod 2), \\
& j \equiv 1(\bmod 2) ; \\
(n-j) k+l+m+i & \text { if } i \equiv 1(\bmod 2), \\
& j \equiv 0(\bmod 2) ; \\
(n+j-1) k+2(l+m)+i+1 & \text { if } i \equiv 1(\bmod 2), \\
& j \equiv 1(\bmod 2) ; \\
(n+j) k+2(l+m)-i+2 & \text { if } i \equiv 0(\bmod 2), \\
j & \equiv 0(\bmod 2) .
\end{array}\right.
$$

It is easy to see that the set of edge weights of $G$ under the labeling $f$ constitute the following set of $q$ odd consecutive integers $\{1,3,5, \ldots, 2 q-1\}$, where $2 q-1=2(n k+m+l)+1$. Hence $f$ is an odd graceful labeling of G.

Theorem $2.3 C b_{n}(2,3, \ldots, n+1) \cup K_{1, m}$ have odd graceful labeling for $n, m \geq 2$.

Proof. Consider $G \cong C b_{n}(2,3, \ldots, n+1) \cup K_{1, m}$. We have $|V(G)|=\frac{(n+1)(n+2)}{2}+m+1$

And $q=|E(G)|=\frac{n(n+3)}{2}+m$, where

$$
V(G)=\left\{x_{i, j}: 1 \leq i \leq j+1,0 \leq j \leq n\right\} \cup\left\{y_{i}: 0 \leq i \leq m\right\}
$$

And

$$
\begin{aligned}
& E(G)=\left\{x_{1, j} x_{1, j+1}: 0 \leq j \leq n-1\right\} \cup \\
& \left\{x_{i, j} x_{i+1, j}: 1 \leq i \leq j, 1 \leq j \leq n\right\} \cup \\
& \left\{y_{0} y_{i}: 1 \leq i \leq m\right\} .
\end{aligned}
$$

To show that $\mathrm{G}$ is odd graceful we define the labeling

$$
f: V(G) \rightarrow\{0,1,2, \ldots, 2 q-1\} .
$$

For $0 \leq i \leq m$ 


$$
f\left(y_{i}\right)= \begin{cases}\left\lfloor\frac{n^{2}+4(n)}{2}\right\rfloor+1 & \text { if } i=0 \\ \left\lfloor\frac{n^{2}+4(n)}{2}\right\rfloor+2 i-1 & \text { if } 1 \leq i \leq m .\end{cases}
$$

Case $(i)$

For $n, m \geq 2$ and even $n$

$$
f\left(x_{1,0}\right)=\left\lfloor\frac{n^{2}+4 n}{2}\right\rfloor
$$

For $1 \leq i \leq j+1,1 \leq j \leq n$

$$
f\left(x_{i, j}\right)=\left\{\begin{array}{lr}
\left\lfloor\frac{n^{2}+4 n}{2}\right\rfloor-\frac{j^{2}+4 j-5}{2}+i-4 & \text { if } i \equiv 0(\bmod 2), \\
\left\lfloor\frac{n^{2}+4 n}{2}\right\rfloor-\frac{(j-2)(j+4)}{2}-i-3 & \text { if } i \equiv 1(\bmod 2) ; \\
\left\lfloor\frac{n^{2}+4(n+m)}{2}\right\rfloor+\frac{(j-1)(j+3)}{2}-i & j \equiv 0(\bmod 2) ; \\
& j \equiv 1(\bmod 2), \\
\left\lfloor\frac{n^{2}+4(n+m)}{2}\right\rfloor+\frac{j^{2}-4}{2}+i-1 & \text { if } i \equiv 0(\bmod 2) ; \\
& j \equiv 0(\bmod 2),
\end{array}\right.
$$

Case(ii)

For $m \geq 2, n \geq 3$ and odd $n$

$$
f\left(x_{1,0}\right)=\left\lfloor\frac{n^{2}+4 n}{2}\right\rfloor
$$

For $1 \leq i \leq j+1,1 \leq j \leq n$

$$
f\left(x_{i, j}\right)=\left\{\begin{array}{lr}
\left\lfloor\frac{n^{2}+4 n}{2}\right\rfloor-\frac{j^{2}+4 j-5}{2}+i-4 & \text { if } i \equiv 0(\text { mod }), \\
\left\lfloor\frac{n^{2}+4 n}{2}\right\rfloor-\frac{(j-2)(j+4)}{2}-i-3 & \text { if } i \equiv 1(\text { mod }), \\
\left.\left\lfloor\frac{n^{2}+4(n+m)-3}{2}\right\rfloor+\frac{(j-1)(j+3)}{2}-i+1\right) & j \equiv 0(\text { mod }) ; \\
& j \equiv 1(\text { mod }), \\
\left\lfloor\frac{n^{2}+4(n+m)+1}{2}\right\rfloor+\frac{j^{2}-4}{2}+i & j \equiv 1(\text { mod }) ; \\
& j \equiv 0(\text { mod }), \\
& j \equiv 0(\text { mod }) .
\end{array}\right.
$$

The labeling $f$ produce the set of edge weights consisting of $q$ consecutive odd integers i.e., $\{1,3, \ldots, 2 q-1\}$, where $2 q-1=n(n+3)+2 m-1$. Thus $f$ is an odd graceful labeling of $\operatorname{graph}_{G}$.

Theorem $2.4 C b_{n}(2,3, \ldots, n+1) \cup B_{m, l}$ for $m \neq l$ and $l>m$ has odd graceful labeling where $n, m, l \geq 2$.

Proof. Consider $G \cong C b_{n}(2,3, \ldots, n+1) \cup B_{m, l}$.
We have $|V(G)|=\frac{(n+1)(n+2)}{2}+m+l+2$

And

$$
q=|E(G)|=\frac{n(n+3)}{2}+m+l+1,
$$

Where

$$
\begin{aligned}
& V(G)=\left\{x_{i, j}: 1 \leq i \leq j+1,0 \leq j \leq n\right\} \cup\left\{z_{i}: 0 \leq i \leq m\right\} \cup \\
& \left\{y_{i}: 0 \leq i \leq l\right\}
\end{aligned}
$$

And

$$
E(G)=\left\{x_{1, j} x_{1, j+1}: 0 \leq j \leq n-1\right\} \cup\left\{x_{i, j} x_{i+1, j}: 1 \leq i \leq j, 1 \leq j \leq n\right\}
$$

$\cup\left\{z_{0} z_{i}: 1 \leq i \leq m\right\} \cup\left\{y_{0} y_{i}: 1 \leq i \leq l\right\} \cup\left\{z_{0} y_{0}\right\}$.

To show that $G$ is odd graceful we define the labeling $f: V(G) \rightarrow\{0,1,2, \ldots, 2 q-1\}$.

For $n, m, l \geq 2, m \neq l$ and $l>m$

For $0 \leq i \leq m$

$$
f\left(z_{i}\right)= \begin{cases}i & \text { if } 0 \leq i \leq 1 \\ 2 i-1 & \text { if } 2 \leq i \leq m .\end{cases}
$$

For $0 \leq i \leq l$

$$
\begin{gathered}
f\left(y_{i}\right)= \begin{cases}n(n+3)+2(m+l)+1 & \text { if } i=0 \\
2 i & \text { if } 1 \leq i \leq l .\end{cases} \\
f\left(x_{1,0}\right)=\left\lfloor\frac{n^{2}+4 n}{2}\right\rfloor+2 l+1
\end{gathered}
$$

For $1 \leq i \leq j+1,1 \leq j \leq n$

$$
f\left(x_{i, j}\right)=\left\{\begin{array}{l}
\left\lfloor\frac{n^{2}+4 n}{2}\right\rfloor-\left(\frac{j^{2}+4 j-5}{2}\right)+2 l+i-3 \\
\left\lfloor\frac{n^{2}+4 n}{2}\right\rfloor-\frac{(j-2)(j+4)}{2}+2(l-1)-i \\
\left\lfloor\frac{n^{2}+4(l+m+n+1)}{2}\right\rfloor-i+1+\frac{(j-1)(j+3)}{2} \\
\left\lfloor\frac{n^{2}+4(l+m+n+1)}{2}\right\rfloor+\frac{j^{2}-4}{2}+i
\end{array}\right.
$$

if $i \equiv 0(\bmod 2)$, $j \equiv 1(\bmod 2)$; if $i \equiv 1(\bmod 2)$, $j \equiv 0(\bmod 2)$; if $i \equiv 1(\bmod 2)$, $j \equiv 1(\bmod 2)$; if $i \equiv 0(\bmod 2)$, $j \equiv 0(\bmod 2)$.

The set of edge weights of $G$, under the labeling $f$, constitute the following set of consecutive odd integers $\{1,3, \ldots, 2 q-1\}$, where $2 q-1=n(n+3)+2(m+l+1)-1$. This proves $f$ to be an odd graceful labeling of $G$.

\section{Conclusion}

In the present work we investigate new families of odd graceful graphs. To investigate similar results for other graph 
families and in the context of different labeling techniques is an open area of research.

\section{References}

[1] M. Baca, C. Barrientos, Graceful and edge-antimagic labeling, Ars Combin., 96(2010),505-513.

[2] M. Baca, M. Miller, Super Edge-Antimagic Graphs, Brown Walker Press, (2008).

[3] C. Barrientos, Odd-graceful labelings, preprint.

[4] P. Eldergill, Decomposition of the Complete Graph with an Even Number of Vertices, M. Sc. Thesis, McMaster University, 1997.

[5] J.A. Gallian, A dynamic survey of graph labeling, Electron. J. Combinatorics, 17(2014),\#DS6

[6] Z. Gao, Odd graceful labelings of some union graphs, J. Nat.
Sci. Heilongiiang Univ., 24 (2007), 35 -39.

[7] R. B. Gnanajothi, Topics in Graph Theory, Ph. D. Thesis, Madurai Kamaraj University, 1991. S. W. Golomb, How to number a graph, in Graph Theory and Computing, R. C. Read, ed., Academic Press, New York (1972), 23-37.

[8] R. L. Graham and N. J. A. Sloane, On additive bases and harmonious graphs, SIAM J. Alg. Discrete Meth., 1 (1980) 382-404.

[9] A. Riasat, S. javed and S. Kanwal, On odd graceful labeling of disjoint union of graphs, Utilitas Math., In press.

[10] G. Ringel, Problem 25, in Theory of Graphs and its Applications, Proc. Symposium Smolenice 1963, Prague (1964), 162.

[11] A. Rosa, On certain valuations of the vertices of a graph, Theory of Graphs (Internat. Symposium, Rome, July 1966), Gordon and Breach, N. Y. and Dunod Paris (1967), 349-355.

[12] D. B. West, An Introduction to Graph Theory, Prentice-Hall, (1996). 